\title{
Microglial activation - tuning and pruning adult neurogenesis
}

\section{Christine T. Ekdahl*}

Inflammation and Stem Cell Therapy Group, Division of Clinical Neurophysiology, Wallenberg Neuroscience Center, Department of Clinical Sciences, Lund University and Skåne University Hospital, Lund, Sweden

\section{Edited by:}

Roger A. Barker, University of Cambridge and Addenbrooke's Hospital, UK

\section{Reviewed by:}

Bradley D. Pearce, Emory University

School of Medicine, USA

Gerd Kempermann, Center for

Regenerative Therapies, Germany

*Correspondence:

Christine T. Ekdahl, Inflammation and Stem Cell Therapy Group, Division of Clinical Neurophysiology, Wallenberg Neuroscience Center, Department of Clinical Sciences, Lund University and Skåne University Hospital, Sölvegatan 17, SE-221 84 Lund, Sweden. e-mail: christine.ekdahl_clementson@ med.lu.se
New neurons are continuously generated in two adult brain regions: the subgranular zone of the hippocampus and the subependyma by the lateral ventricles, referred to as the neurogenic niches. During their development from neural stem cells to mature functionally integrated neurons numerous choices are made, such as proliferation or quiescence, cell survival or death, migration or establishment, growth or retraction of processes, synaptic assembly or pruning, or tuning of synaptic transmission. The process is altered by physiological stimuli as well as several brain diseases. Microglia are located within the neurogenic niches and have become interesting candidates for modulating neurogenesis in both the healthy and injured brain. They become activated by foreign antigens or changes in the brain homeostasis and transform this innate immunity into an adaptive immune response by recruiting systemic immune cells. Most studies report an acute decrease in the survival of new neurons following this classically activated microglia reaction. The long-term effects are more complex. In neurodegenerative diseases, microglial activation is more heterogeneous and the transformation from a pro- to an anti-inflammatory cytokine profile and the deactivation of microglia is not well defined. The diversity is reflected by numerous reports describing both beneficial and detrimental effects on neurogenesis, primarily on the proliferation, survival, and cell fate. However, relatively few studies have investigated alterations at later stages of neurogenesis including the functional integration. Though likely, it is not established how a fine-tuned cross-talk between microglia and adult-born neurons would work and how it changes upon microglia activation. This review will therefore launch three hypotheses for how microglia might direct synaptic integration of newborn neurons, currently a fast expanding research field.

Keywords: microglia, neurogenesis, synaptic pruning, synaptic transmission, adult-born neurons, subgranular zone, subependyma, neurogenic niche

\section{FROM NEURAL STEM CELLS TO MATURE FUNCTIONALLY INTEGRATED NEURONS - CHOICES TO BE MADE}

Neurogenesis persists in two adult brain regions: the subependyma of the lateral ventricles and the subgranular zone (SGZ) of the hippocampal dentate gyrus. In the subependymal layer, multipotential, self-renewing stem cells are the source of newly generated neurons migrating through the rostral migratory stream and incorporating into the olfactory bulb as interneurons (Seaberg and van der Kooy, 2002). New olfactory neurons are thought to participate in both long-term olfactory memory and predator avoidance (Sakamoto et al., 2011; Sultan et al., 2011). From the SGZ, neuronal and glial progenitors with limited self-renewal capacity migrate into the granule cell layer and develop primarily into granule cells, a few becomes interneurons (Seaberg and van der Kooy, 2002; Liu et al., 2003; Livneh and Mizrahi, 2011). The integration of new hippocampal neurons in the adult brain occurs over several months, with an initial tonic GABA-induced depolarization converting into an intermittent GABA-mediated hyperpolarization when the cells are 3 weeks of age (Ge et al., 2008). Mature excitatory synapses are visualized at about 2 months, while spine density increases in the new neurons up to 6 months of age (Zhao et al., 2006). Simultaneously, axons grow and establish either functional glutamatergic synapses with hilar interneurons, mossy cells and CA3 pyramidal neurons (Toni and Sultan, 2011) or occasionally GABAergic inhibitory synapses with granule cells (Liu et al., 2003). The continuous integration of adult-born hippocampal neurons is important for synaptic transmission and bidirectional plasticity in the dentate gyrus. It is suggested to account for an efficient integration of novel incoming information and in memory formation (Dupret et al., 2008; Massa et al., 2011; Sahay et al., 2011), such as pattern separation and pattern integration (Deng et al., 2010).

The development of adult-born neurons may also be described as a continuous decision process. Neural stem cells/progenitors will have to decide whether to proliferate or stay in quiescence. Almost immediately after birth some cells undergo apoptotic death, while others survive. The surviving cells will choose a neuronal or glial fate and thereafter either stay and establish contacts within the SGZ or subependyma, the so-called neurogenic niches, or migrate into other brain regions. The integration of new neurons includes both growth and retraction of dendrites and axons, 
assembly and pruning of excitatory and inhibitory synapses, and homeostatic tuning of the established synaptic transmission. It becomes evident that in order for the new neurons to make the most appropriate choices for the overall function of the surrounding network, they are dependant on environment cues at most likely all developmental stages.

\section{THE NEUROGENIC NICHE}

It is conceivable that the local circuitries within the adult neurogenic niches have region-specific instructive roles in directing neuronal production and stem cell maintenance. It has even been suggested that they may shield ongoing neurogenesis from possible external inhibitory influences (Riquelme et al., 2008). The neurogenic niches consists of several cell types and structures, including: (1) astrocytes that envelop and contact all cell types and structures in the niche, secret diffusible signals, and form a gap junctiondependant syncytium by which they propagate signals and may regulate activation and differentiation of stem cells. They also act as neural stem cells/precursors (Riquelme et al., 2008), (2) ependymal cells by the subependymal layer that regulate the absorption of ions, transport factors from the cerebral spinal fluid into the parenchyma, and act as a source of secreted pro-neurogenic factors like transforming growth factor (TGF)- $\alpha$ and basic fibroblast growth factor (Riquelme et al., 2008), (3) blood vessels that undergo a parallel angiogenesis within the niche and often use common factors with neurogenesis such as vascular endothelial growth factor, nitric oxide and erythropoietin (Riquelme et al., 2008), (4) meningeal projections, extracellular matrix (ECM) proteins, basal lamina, and perivascular cells and fibroblasts, which propagate or modulate signals from the blood vessels and cerebral spinal fluid as well as from surrounding brain parenchyma. Emerging evidence underscore the important interaction between the adult-born neurons and ECM proteins. Cell adhesion to its environment includes matrix components, extracellular proteolytic enzymes, integrins, and non-receptor tyrosine kinases, which influence both gene expression and post-transcriptional signaling cascades (Riquelme et al., 2008; Wojcik-Stanaszek et al., 2011), (5) interneurons and other neighboring mature neurons already highly integrated into the hippocampal network, which may modulate neurogenesis by activity- and signaling-dependent mechanisms (Markwardt et al., 2011; Masiulis et al., 2011; Toni and Sultan, 2011), (6) oligodendrocytes, which are numerous in the subependyma but few in the SGZ. Their interaction with the neural progenitor cells is, however, so far unclear (Morrens et al., 2012), and (7) myeloid cells including microglia and dendritic cells, which are located in close proximity to the newborn neurons, where their activity correlates with the neurogenic response (Ekdahl et al., 2003; Monje et al., 2003; Bulloch et al., 2008; Sierra et al., 2010).

\section{MICROGLIAL ACTIVATION IN THE ADULT BRAIN}

A microglia activation profile is constantly modulated by either initiating factors, such as pathogen- or damage-associated molecular patterns (Schratt et al., 2006; Aronica and Crino, 2011) or alterations in electromagnetic fields (Richerson et al., 2005), or intracellular transducing signals, or feed-forward loops amplifying resolution mechanisms, or feed-back loops as counter-regulators
(Glass et al., 2010). During brain pathology, the microglial activation is often divided into two phenotypic profiles: the classical M1 and the alternative M2 activation/deactivation state (Gordon, 2003; Mosser, 2003; Michelucci et al., 2009). The classical M1 activation occurs as microglia encounter a foreign antigen. The microglia act as a first line of defense and participate in transforming the innate immunity into an adaptive immune response by recruiting systemic immune cells. The alternative and deactivated M2 phenotype, sometimes called "neuroprotective," is important when switching from a classical inflammatory response to a reduction of pro-inflammatory mediators, an increased production and release of anti-inflammatory cytokines, neurotrophic factors, and a production of cytoactive factors involved in repair and restructuring of the damaged ECM in the brain. However, these M2 phenotypes may also participate in chronic "neuroinflammation" in the brain (Colton, 2009). The heterogeneity becomes evident in for instance neurodegenerative diseases, where the transformation from a pro- to an anti-inflammatory cytokine profile and the deactivation of microglia is neither temporally nor spatially clearly defined. There are even suggestions for a dysfunctional microglia phenotype following long-lasting activation (Graeber, 2010).

In the healthy brain, microglia and/or perivascular cells have been suggested to form an "immunological blood-brain barrier" between the brain parenchyma and the vascular system. However upon activation, by for instance sterile inflammation following trauma, ischemia, or chemical damage, these cells may instead recruit blood-born neutrophils and macrophages and increase the amount of tissue damage (Perry, 2010; Rock et al., 2010; Graeber et al., 2011; Yirmiya and Goshen, 2011). Simultaneously, microglia seems to be able to protect neurons from systemic immune cells by direct engulfment of invading neutrophils (Neumann et al., 2008). Other circulating immune cells are, though, required to maintain and sense brain homeostasis through the choroid plexi, brain meninges and the cerebral blood fluid. For instance, recruitment of systemic circulating $\mathrm{T}$ cells specific to CNS antigens can promote the termination of a local neurotoxic inflammatory response together with microglia of a "neuroprotective" phenotype (Ziv et al., 2006; Shechter et al., 2009; Ron-Harel et al., 2011). Taken together, these findings suggest that the activation state of the microglia is closely intermingled with the alertness of systemic immune cells and that there may be a bidirectional cross-talk.

\section{HETEROGENEOUS MICROGLIAL POPULATION WITHIN THE NEUROGENIC NICHES - REGION SPECIFICITY?}

Microglial density and phenotype differ between regions of the healthy brain, which could implicate functional differences and sensitivity to the surrounding environment (Lawson et al., 1990; Olah et al., 2011). Also within the subependymal layer of the healthy adult rat brain, microglia constitute a heterogeneous cell population. Here, the vast majority of ionized calcium-binding adapter molecule 1 (Iba1)-immunostained microglia exhibit a ramified or surveying phenotype, followed by an intermediate form with shorter processes, less arborization, and larger soma (Thored et al., 2009). Few express an amoeboid or round morphology (Figures 1A-D). There are partly overlapping subpopulations of microglia expressing the phagocytic marker ED1, 
associated with a pro-inflammatory profile, as well as major histocompatibility complex (MHC) class II, insulin-like growth factor-1 (IGF-1), and triggering receptor expressed on myeloid cells-2, the three latter molecules being associated with alternative or anti-inflammatory microglia activation (Thored et al., 2009; Heldmann et al., 2011). Local proliferation of microglia occurs, but parts of the subependymal microglia population are also blood-born (Thored et al., 2009; Heldmann et al., 2011). Interestingly, following middle cerebral artery occlusion microglia develop different morphological phenotypes within the subependymal layer compared to the striatal peri-infarct area. The differences are evident at least 16 weeks post-injury, including fewer amoeboid and round microglia morphologies and an up-regulation of IGF-1 in the subependyma. This may imply a region-specific, permissive microglia population within the neurogenic niche (Thored et al., 2009). However, a regionspecific depletion of parts of the microglia population (expressing CD11b/Mac-1) within the subependyma, early after an ischemic lesion in rats, did not alter the number of newly formed neuroblasts in the striatum or their migratory distance (Heldmann et al., 2011).

In the hippocampal SGZ of rodents housed in an enriched environment, increased number of Iba1+ microglia co-labeled with the anti-inflammatory factors IGF-1 and MHC class II, were found together with $\mathrm{T}$ cell recruitment and an increase in neurogenesis. Moreover, $\mathrm{T}$ cell deficient mice showed reduced neurogenesis, which could not be overcome by an enriched environment ( $\mathrm{Ziv}$ et al., 2006). The findings suggest a beneficial role of SGZ microglia possibly instructed by CNSspecific $\mathrm{T}$ cells. Conversely, following seizures and in neurodegenerative models of Alzheimer's disease, an increased number of Iba1+ microglia expressing ED1 is evident both acutely and chronically, and primarily cytotoxic for the new neurons (Ekdahl et al., 2003; Bonde et al., 2006; Biscaro, Lindvall, Tesco, Ekdahl, Nitsch, unpublished observation). Taken together, microglia are capable of developing both pro- and anti-inflammatory activation profiles in neurogenic brain areas. Moreover, it is possible that under certain circumstances the temporal and spatial constitution of the activation may even be region-specific.

Apart from the heterogeneous morphology and plethora of immune mediators, the motility and dynamic configurations of the microglia are also likely to reflect different functional aspects. Microglial processes are highly mobile and continuously rebuilt. Two-photon imaging of neocortex has shown active microglia continually surveying their microenvironment even in the normal brain (Nimmerjahn et al., 2005). The motility of microglia and perhaps region-specific features within the neurogenic niches remains to be characterized.

\section{MICROGLIA - BENEFICIAL AND DETRIMENTAL FOR NEUROGENESIS}

In the last two decades many studies have implicated a central role for brain parenchymal microglia as well as systemic immune cells during the generation of adult-born neurons (Ekdahl et al., 2009; Whitney et al., 2009; Molina-Holgado and Molina-Holgado, 2010; Russo et al., 2011a). Among the first evidence for a beneficial role of microglia or microglia-released mediators are in vitro studies on neural stem cell cultures, where the formation of neuroblasts could be rescued by co-culturing with microglia or conditioned media from microglia (Aarum et al., 2003; Walton et al., 2006). This "pro-neurogenic" function of microglia has since then been further supported by a number of studies from the group of $\mathrm{M}$. Schwartz. Originally, they demonstrated that microglia activated with cytokines related to $\mathrm{T}$ cell helper cells promoted neurogenesis in vitro (Butovsky et al., 2006) and that CNS-specific $\mathrm{T}$ helper cells regulate hippocampal neurogenesis in vivo (Ziv et al., 2006). Interestingly, mice devoided of $\mathrm{T}$ cells or both $\mathrm{T}$ and $\mathrm{B}$ cells showed impairments in several hippocampal-dependent spatial learning tests (Kipnis et al., 2004; Brynskikh et al., 2008; RonHarel et al., 2008), which could be improved by replenishing $\mathrm{T}$ cells or boosting $\mathrm{T}$ cell activation by agonist of self-reactive $\mathrm{T}$ cells (Kipnis et al., 2004). Even more intriguingly was the finding that cognitive tasks increased the number of $\mathrm{T}$ cells located within the meningeal spaces, where $\mathrm{T}$ cell depletion induced a proinflammatory phenotype of the myeloid cells (Derecki et al., 2010). Since neurogenesis is thought to be important for hippocampal plasticity and memory formation, the health of the systemic immune system could possibly directly or through the interaction with innate non-neuronal cell types, like microglia, continuously shape neuroplasticity in both the healthy and damaged brain. Another recent reports on the beneficial role of microglia for newly formed neurons comes from Bachstetter et al. (2011) who have studied the neuronal transmembrane chemokine fractalkine (FKN)/CX3CL1 and its microglia-expressed receptor, CX3CR1pathway. Disruption of this pathway in young adult rodents decreased both survival and proliferation of hippocampal neural progenitor cells (Bachstetter et al., 2011). Unchallenged microglia have also been demonstrated to engulf apoptotic bodies of neural progenitors in the SGZ. During normal neurogenesis in the healthy brain, a large proportion of neural progenitors undergo apoptosis within their first days in life. By rapidly removing cell debri, the scavenging properties of microglia could have an important regulator role at the early stages of neurogenesis (Sierra et al., 2010). However, it is not fully clear whether the phagocytosis is beneficial for the surrounding newborn neurons by reducing pro-inflammatory mediators, or may be detrimental by further inducing apoptotic neuronal death (Magnus et al., 2001; Neher et al., 2011).

The first studies suggesting an acute detrimental role of activated microglia for neurogenesis were performed in rats following intraparenchymal lipopolysaccharide (LPS) injections (Ekdahl et al., 2003; Monje et al., 2003). LPS-induced microglial activation led to a dramatic decrease in the survival of newly formed hippocampal neurons. The pivotal role of microglial activation was further substantiated following both epileptic seizures and irradiation, where hippocampal neurogenesis could be rescued by inhibiting microglia activation through either administration of the anti-inflammatory agent minocycline or nonsteroidal anti-inflammatory drugs (Ekdahl et al., 2003; Monje et al., 2003). Pro-inflammatory cytokines like interleukin (IL)1, IL-6, and tumor necrosis factor (TNF), released acutely by activated microglia, have been suggested as important mediators between the microglia and the new neurons (Monje et al., 2003; 

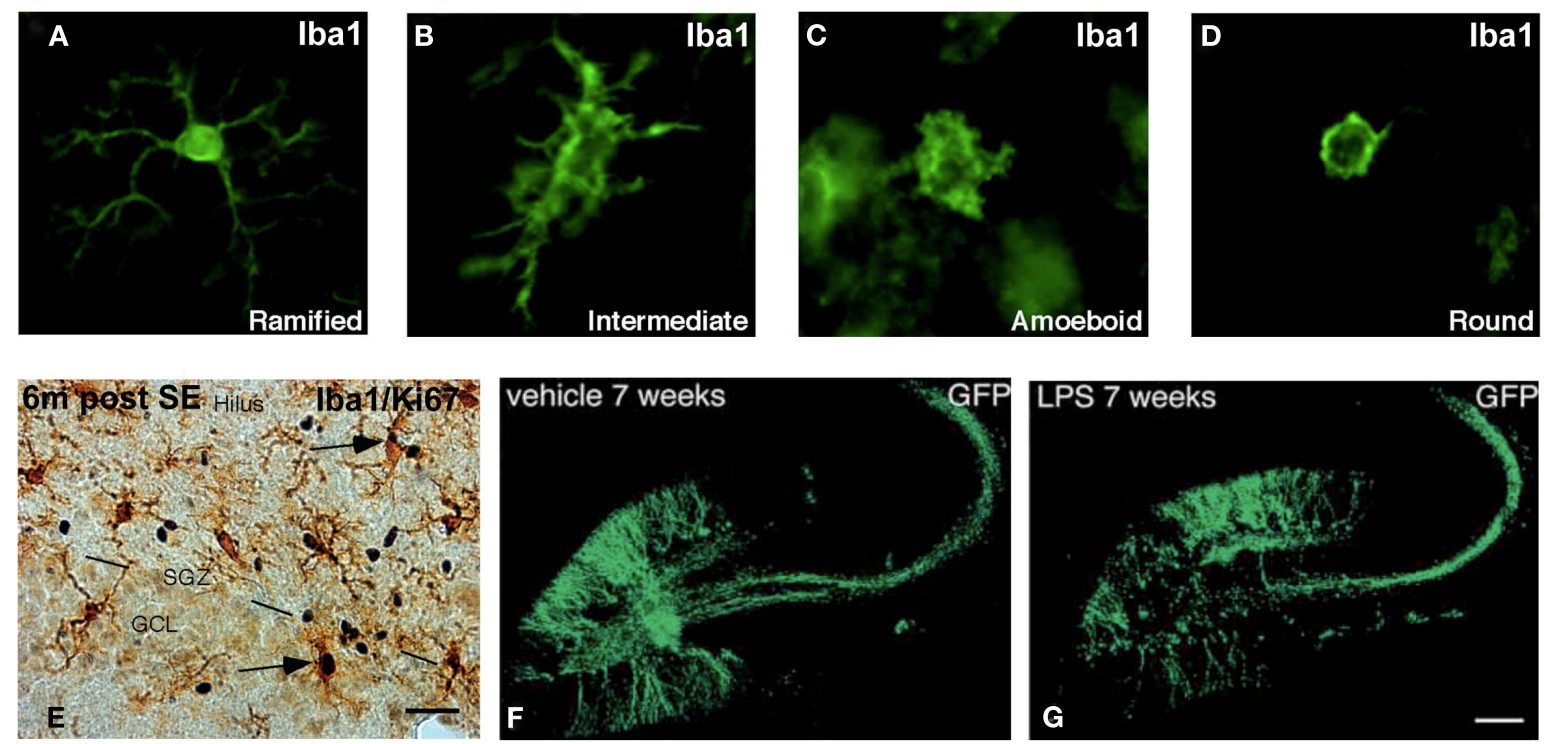

FIGURE 1 | Microglia and newly generated neurons in the neurogenic niche of the adult rat brain. (A-D) Photomicrographs showing four distinct phenotypes of Iba1+ microglia. (E) Image of Iba1+ microglia within the subgranular zone (SGZ) of the hippocampal dentate gyrus 6 months after electrically induced status epilepticus. Arrows mark microglia that express Ki-67, which implicate an ongoing proliferation. (F,G) Retroviral-GFP vector labeling of 7-week-old adult-born neurons in the hippocampus following vehicle or LPS-induced microglial activation. The cell bodies of the newly generated neurons are located in the SGZ and granule cell layer of the dentate gyrus. Their dendritic trees are visible throughout the molecular layer and their axons extend into the dentate hilus and the CA3 region. Modified from Bonde et al. (2006), Jakubs et al. (2008), and Thored et al. (2009).
Iosif et al., 2006; Yirmiya and Goshen, 2011). Also prostaglandins released by the microglia may negatively regulate different steps of neurogenesis. In support, mice lacking cyclooxygenase-1 lack both the acute LPS-induced decrease in proliferation, survival, and differentiation of new hippocampal neurons (Russo et al., 2011b).

When the initiating agent for microglia activation is no longer present and the acute pro-inflammatory phase is over, an antiinflammatory cytokine profile may develop, as shown after LPSstimulation (Cacci et al., 2008). This profile may be beneficial for the newly formed neurons, since for instance administration of anti-inflammatory cytokines like TGF- $\beta$, has provided beneficial effects on neurogenesis in the subependyma (Mathieu et al., 2010a). However, if the microglia are continuously activated, they may partly sustain their release of oxidative stressors, which could be neurotoxic (Glass et al., 2010; Nathan and Ding, 2010; Polazzi and Monti, 2010). The result may be disease-specific interaction between microglia and the newborn neurons (Graeber et al., 2011). Microglia are, though, important for terminating immune/immune-like responses, by, i.e., recruitment of systemic immune cells (Frank-Cannon et al., 2009; Shechter et al., 2009; Polazzi and Monti, 2010; Rivest, 2011; Ron-Harel et al., 2011), and may thereby protect new neurons and even recruit neural progenitors for repair (Czeh et al., 2010; Mathieu et al., 2010b). The correlation between seizure-induced microglial activation and neurogenesis is a typical example of this complexity. A severe seizure insult induces a strong transient increase in hippocampal neurogenesis (Parent et al., 1997), followed by a chronic decrease below control levels (Hattiangady and Shetty, 2010). In the acute phase, microglia activation is prominent within the SGZ and has been suggested to compromise the early survival of the new neurons (Ekdahl et al., 2003). Six months later, the microglial activation is decreased but still present (Bonde et al., 2006; Figure 1E). This may imply also a long-term negative correlation between the production of new neurons and the chronically activated microglia. However, the newly formed neurons generated directly after the seizure insult, that survived the acute post-seizure environment, are still present 6 months later. They have even been estimated to comprise about $10 \%$ of the total dentate granule cell layer. These adult-born neurons are surrounded by the chronically activated microglia, which may instead favor a possible supportive role of the chronically activated microglia (Bonde et al., 2006). Because the initial seizure insult is followed by additional spontaneous seizures throughout the life of the animals, there is a possibility for recurrent triggering of the microglial activation. Whether subpopulations of microglia with either detrimental or beneficial effect on the surrounding newborn neurons co-exist within the neurogenic niche is not known and may be directly depending on the number of spontaneous seizures. The initial severity of the seizure insult is directly correlated to both the number of activated microglia as well as the neurogenic response (Mohapel et al., 2004). In addition, parts of the newly generated neurons migrate aberrantly out into the dentate hilus, which may perhaps be due to the well-known seizure-induced death of hilar interneurons and the prominent population of activated hilar microglia. Collectively, these findings support a primarily beneficial interaction between microglia and new neurons in the intact brain. However, the cross-talk is complex and probably double-edged in pathological conditions, especially following long-term microglial activation. 


\section{THE ROLE OF MICROGLIA DURING FUNCTIONAL INTEGRATION OF ADULT-BORN NEURONS - AN EQUALIZER AMPLIFYING AND FILTERING SYNAPTIC SIGNALING?}

Until now, most studies on the cross-talk between microglia and adult-born neurons have focused on the effect on the early stages of neurogenesis, such as proliferation, survival, and neuronal fate. The role of microglia at later stages of neurogenesis, i.e., during synaptic assembly, stability, and transmission is less characterized. Today, there is no direct evidence that microglia could regulate synaptic integration of adult-born neurons. However, there is some evidence and several suggestions that microglia regulate synaptic pruning and transmission in mature neurons, currently a fast expanding research area. Three main working hypotheses can be put forward for how microglia may also regulate synaptic integration of adult-born neurons: (1) involvement of microglia in synaptogenesis and pruning, (2) modulation of perisynaptic structures, and (3) spine structure and synaptic transmission.

\section{MICROGLIA MAY BE INVOLVED IN SYNAPTOGENESIS AND PRUNING OF SYNAPSES ON ADULT-BORN NEURONS}

This hypothesis is based on recent studies describing how microglia may be involved in synaptic elimination/stripping/pruning by phagocytic engulfment of synapses on mature neurons in the healthy brain (Paolicelli et al., 2011; Tremblay and Majewska, 2011). By electron microscopy and twophoton in vivo imaging of the primary visual cortex of juvenile mice during visual manipulations, subtle changes in the behavior of quiescent microglia were observed. This included geometric regulation of perisynaptic extracellular spaces, contact with subsets of structurally dynamic and transient dendritic spines, and phagocytic engulfment of intact synapses (Tremblay et al., 2010; Tremblay and Majewska, 2011). The findings were further substantiated by Paolicelli et al. (2011) proposing synaptic pruning by microglia during postnatal development in mice. Mice lacking microglia expressing the chemokine FKN receptor CX3CR1, exhibited a transient reduction in microglia number correlated with a delayed synaptic pruning. This resulted in an excess of dendritic spines and immature electrophysiological properties of CA1 pyramidal neurons at P13 and P16 in the CX3CR1 knockout mice (Paolicelli et al., 2011). The occurrence of synaptic pruning has also been correlated with the duration of the microglia-synaptic contacts. In vivo imaging studies of the ischemic brain, suggest more persistent contacts between microglia processes and dendritic spines and axon terminals, compared to the transient $4-5 \mathrm{~min}$ of normal contacts observed in the healthy brain. Following longer interactions in the pathological environment, these contacted synapses often disappeared (Wake et al., 2009; Kettenmann et al., 2011). In several brain pathologies, such as following axonal lesions or immune mediated-cortical lesions, pruning of synapses from the perikaryon and dendrites is evident. The suggested consequence of synaptic pruning during these conditions has been neuroprotection (Cullheim and Thams, 2007; Trapp et al., 2007; Kettenmann et al., 2011).

An interesting possible signaling pathway between the microglia and the synaptic structures on the new neurons is the complement cascade. Since the complements are involved in opsonization and cytolysis, they have become attractive possible candidates for executing synaptic pruning in both the healthy and injured brain. Mice deficient in the initiating protein in the classical complement cascade, C1q, exhibit large sustained defects in synapse elimination, excessive excitatory synapses and axon terminals in mature neurons (Stevens et al., 2007; Chu et al., 2010). Neural progenitor cells and immature neurons express the receptors for complement fragments $\mathrm{C} 3 \mathrm{a}$ and $\mathrm{C} 5 \mathrm{a}$. C3a stimulates neuronal migration and differentiation, by modulating stromal cell-derived factor-1a-induced extracellular-signal-regulated kinases phosphorylation. Mice lacking C3 signaling (including $\mathrm{C} 3 \mathrm{a}$ and $\mathrm{C} 5 \mathrm{a}$ ) have reduced basal neurogenesis as well as decreased survival of ischemia-generated new striatal neurons (Rahpeymai et al., 2006; Shinjyo et al., 2009). Also complement receptor 2 ( Cr2) is expressed in adult neural progenitor cells from the dentate gyrus, though, Cr2 knockout mice exhibit increased hippocampal neurogenesis (Moriyama et al., 2011). Together these results suggest a fundamental role of the complements for neurogenesis, including proper synaptic pruning and integration of newly formed neurons. However, in a mouse model of glaucoma, unwanted synapses may be tagged by complement for elimination, which suggests that complement-mediated synaptic pruning can become aberrantly reactivated in neurodegenerative disease (Stevens et al., 2007). In human temporal lobe epilepsy with hippocampal sclerosis, the complements factors are expressed in both astrocytes, mature neurons and microglia, but particularly in microglia (Aronica et al., 2007). Whether it is really the microglia that eliminate the complement-tagged neuronal synaptic terminals on either mature or newly formed neurons, in the intact and the damaged brain, is not yet shown (Perry and O'Connor, 2008; Tremblay and Majewska, 2011). Another suggested pathway between microglia and newborn neurons is the MHC class I and its related receptors, which is expressed in both microglia and in neurospheres and the subependymal layer (Popa et al., 2011). MHC class I molecules have been shown to influence both the strength and pattern of synaptic elimination (Cullheim and Thams, 2007; Kettenmann et al., 2011).

\section{MICROGLIA MAY MODULATE THE PERISYNAPTIC STRUCTURE OF SYNAPSES ON ADULT-BORN NEURONS}

Microglia might affect synaptic transmission though proteolytic modification of the perisynaptic environment (Tremblay and Majewska, 2011). This would include inactivation, degradation, and/or activation of the ECM, leading to compartmentalization of proteases, ions, cytokines, and neurotransmitters within individual synapses. One interesting possible pathway is through the matrix metalloproteases (MMP), such as MMP-9, shown already to be involved in the migration and differentiation of adult neural progenitor cells in the subependymal layer (Barkho et al., 2008) Another candidate is via a disintegrin and metalloproteases (ADAMs), implicated in dopamine-induced release of epidermal growth factor from stem cells in the subventricular zone (O'Keeffe and Barker, 2011). Proteolytic remodeling of the ECM by MMPs may convert trophic factors to their biologically active forms (i.e., vascular endothelial growth factor and TGF- $\beta$ ) and thereby influence synaptic integration. ECM ligands, such as integrin receptors, 
provide outside-in signals for cells to sense their microenvironment. This leads to modulation of receptor tyrosine kinases, which closely cooperate with growth factors (Wojcik-Stanaszek et al., 2011).

\section{MICROGLIA MAY MODULATE THE STRUCTURE OF DENDRITIC SPINES AND SYNAPTIC TRANSMISSION IN ADULT-BORN NEURONS}

Another mechanism by which microglia may regulate neuronal activity is through remodeling of dendritic spine morphology, including size, shape, and motility (Tremblay and Majewska, 2011), as well as synaptic adhesion and transmission. The dendritic structure is closely associated with its synaptic activity and activity-driven changes in synaptic efficacy may modulate spine morphology due to alterations in the underlying actin cytoskeleton (Fortin et al., 2011).

There are also several immune mediators involved in synaptic remodeling of mature neurons. For instance, in cultured hippocampal neurons, TNF receptor-associated protein 1 knockdown modulated the morphology of dendritic spines (Kubota et al., 2009). TNF deficient mice displayed also smaller dendritic trees in the hippocampus (McCoy and Tansey, 2008). Furthermore, mice lacking IL-1 $\beta$ receptor had reduced dendritic spine size (Goshen et al., 2009) and IL-6 over-expression in cerebellar granule cells caused impairments in granule cell adhesion, migration, and increased formation of excitatory synapses on granule cells (Wei et al., 2011). MicroRNAs, which may control the expression of hundreds of genes (Schratt et al., 2006), were recently suggested to be interacting with immune mediators. Knocking down miR132 in PC12 cells resulted in an increased expression of especially pro-inflammatory molecules. Interestingly, retroviral knockdown of miR-132 impaired the integration of newborn neurons in the adult hippocampus. (Luikart et al., 2011). However, whether or not the structural synaptic changes in either mature or newly formed neurons directly depend on cytokines released by the microglia is not clear.

When synapses have been established, microglia might finetune the synaptic strength to ensure proper synaptic transmission and plasticity (Beique et al., 2011). Mice lacking CX3CR1 show alterations in both hippocampal long-term potentiation (LTP) and long-term depression (LTD), supporting a microglia-driven neuronal plasticity during development and in mature neurons (Bachstetter et al., 2011; Maggi et al., 2011; Paolicelli et al., 2011). In addition, LPS-activated microglia in acute mice hippocampal slices enhance the frequencies of excitatory postsynaptic currents in mature neurons. This effect was abolished by applying purinergic antagonists, especially against the P2Y1 receptor, which is only expressed on interneurons and astrocytes in the hippocampus. By producing ATP, microglia may thereby act on astrocytes through purinergic signaling, which could amplify the ATP production, release glutamate, and act on mature neuronal glutamatergic receptors (Tremblay et al., 2011). Moreover, prolonged changes in electrical activity may lead to uniform adjustments in the strength of all synapses, called homeostatic synaptic scaling/tuning (Stellwagen and Malenka, 2006). Synaptic scaling in response to prolonged blockade of neuronal activity in vitro can be mediated by TNF- $\alpha$ from glial cells (astrocytes and/or microglia; Stellwagen and Malenka, 2006). Also, TNF- $\alpha$ may increase the expression of
AMPA receptors on synapses (Pickering et al., 2005), resulting in excitatory synaptic scaling, and decrease GABAA receptor expression (McCoy and Tansey, 2008). When adult-born hippocampal neurons integrate into a pathological environment, hosting either seizure- (Jakubs et al., 2006) or LPS-induced microglial activation (Jakubs et al., 2008; Figures 1F,G), they possess an increased inhibitory synaptic drive onto their afferent synapses, compared to new neurons formed in healthy conditions. Conversely, in a less severe seizure-environment, without a prominent microglial activation, this increase is lacking (Wood et al., 2011). How or whether it is really the microglial activation that account for these changes observed in the synaptic transmission of newborn neurons needs further investigations, though, it is a tempting speculation.

Microglia may also regulate synaptic transmission in newly formed neurons by directly targeting synaptic adhesion molecules. Seizure-induced microglial activation is associated with structural changes in dendrite spine formation (Murphy et al., 2011), as well as altered expression of synaptic adhesion molecules and scaffolding proteins on newly formed hippocampal neurons (Jackson, Chugh, Nilsson, Karlström, Lindvall, Ekdahl, unpublished observation). In support, several adhesion molecules have been shown to modulate synaptic integration of newborn neurons. One of the most studied adhesion molecules is the polysialated neural cell adhesion molecule (NCAM), which is specifically expressed on new neurons (Gascon et al., 2010). Another interesting pathway is the $\mathrm{N}$-cadherin/beta-catenin/neurogenin signaling cascade, which has been shown to modulate adhesion, neuronal differentiation, and neurite outgrowth in neurospheres (Chen et al., 2006). Also the adhesion molecules neuroligin 3 and 4 are involved in presynaptic differentiation and synapse formation in human induced pluripotent stem cell-derived neurons (Kim et al., 2011). The adhesion molecules may be regulated by immune mediators released by microglia, such as TNF. A recent report described reduced expression of the $\mathrm{N}$-cadherin by TNF receptor-associated protein 1 knockdown in cultured hippocampal neurons (Kubota et al., 2009).

\section{CONCLUDING REMARKS}

Various brain pathologies and physiological stimulations modify neurogenesis (Zhao et al., 2008). Two of the strongest modulators include epileptic seizures involving the SGZ of the dentate gyrus (Bengzon et al., 1997; Parent et al., 1997; Jakubs et al., 2006) and ischemia close to the subependymal layer (Arvidsson et al., 2002). Understanding the signaling pathways between the new neurons and the environment within the neurogenic niches in pathological conditions, may yield new targets for therapeutic interventions. Several drugs are currently studied in order to increase the neuroprotective functions of microglia or to shift the microglial phenotype toward neuroprotection (Polazzi and Monti, 2010). However, microglia in particular may exhibit strong compensatory mechanisms upon external modulation and quickly rearrange their proliferation capacity and phenotypic characteristics. The heterogeneity of the microglial activation makes predictions of the outcome from immune-modulating therapies very difficult (Rivest, 2011). Future studies on the impact of more subtle tunings of the cross-talk between microglia and the newly formed neurons may perhaps ease these interventions. 


\section{REFERENCES}

Aarum, J., Sandberg, K., Haeberlein, S. L., and Persson, M. A. (2003). Migration and differentiation of neural precursor cells can be directed by microglia. Proc. Natl. Acad. Sci. U.S.A. 100, 15983-15988.

Aronica, E., Boer, K., van Vliet, E. A., Redeker, S., Baayen, J. C., Spliet, W. G., van Rijen, P. C., Troost, D., da Silva, F. H., Wadman, W. J., and Gorter, J. A. (2007). Complement activation in experimental and human temporal lobe epilepsy. Neurobiol. Dis. 26, 497-511.

Aronica, E., and Crino, P. B. (2011). Inflammation in epilepsy: clinical observations. Epilepsia 52(Suppl. 3), 26-32.

Arvidsson, A., Collin, T., Kirik, D., Kokaia, Z., and Lindvall, O. (2002). Neuronal replacement from endogenous precursors in the adult brain after stroke. Nat. Med. 8, 963-970.

Bachstetter, A. D., Morganti, J. M., Jernberg, J., Schlunk, A., Mitchell, S. H., Brewster, K. W., Hudson, C. E., Cole, M. J., Harrison, J. K., Bickford, P. C., and Gemma, C. (2011). Fractalkine and CX 3 CR1 regulate hippocampal neurogenesis in adult and aged rats. Neurobiol. Aging 32, 2030-2044.

Barkho, B. Z., Munoz, A. E., Li, X., Li, L., Cunningham, L. A., and Zhao, X. (2008). Endogenous matrix metalloproteinase (MMP)-3 and MMP9 promote the differentiation and migration of adult neural progenitor cells in response to chemokines. Stem Cells 26, 3139-3149.

Beique, J. C., Na, Y., Kuhl, D., Worley, P. F., and Huganir, R. L. (2011). Arcdependent synapse-specific homeostatic plasticity. Proc. Natl. Acad. Sci. U.S.A. 108, 816-821.

Bengzon, J., Kokaia, Z., Elmer, E., Nanobashvili, A., Kokaia, M., and Lindvall, O. (1997). Apoptosis and proliferation of dentate gyrus neurons after single and intermittent limbic seizures. Proc. Natl. Acad. Sci. U.S.A. 94, 10432-10437.

Bonde, S., Ekdahl, C. T., and Lindvall, O. (2006). Long-term neuronal replacement in adult rat hippocampus after status epilepticus despite chronic inflammation. Eur. J. Neurosci. 23, 965-974.

Brynskikh, A., Warren, T., Zhu, J., and Kipnis, J. (2008). Adaptive immunity affects learning behavior in mice. Brain Behav. Immun. 22, 861-869.

Bulloch, K., Miller, M. M., Gal-Toth, J., Milner, T. A., Gottfried-Blackmore, A., Waters, E. M., Kaunzner, U. W., Liu, K., Lindquist, R., Nussenzweig, M. C., Steinman, R. M., and McEwen, B. S. (2008). CD11c/EYFP transgene illuminates a discrete network of dendritic cells within the embryonic, neonatal, adult, and injured mouse brain. J. Comp. Neurol. 508, 687-710.

Butovsky, O., Ziv, Y., Schwartz, A., Landa, G., Talpalar, A. E., Pluchino, S., Martino, G., and Schwartz, M. (2006). Microglia activated by IL-4 or IFN-gamma differentially induce neurogenesis and oligodendrogenesis from adult stem/progenitor cells. Mol. Cell. Neurosci. 31, 149-160.

Cacci, E., Ajmone-Cat, M. A., Anelli, T., Biagioni, S., and Minghetti, L. (2008). In vitro neuronal and glial differentiation from embryonic or adult neural precursor cells are differently affected by chronic or acute activation of microglia. Glia 56, 412-425.

Chen, J., Zacharek, A., Li, Y., Li, A., Wang, L., Katakowski, M., Roberts, C., Lu, M., and Chopp, M. (2006). $\mathrm{N}$-cadherin mediates nitric oxideinduced neurogenesis in young and retired breeder neurospheres. $\mathrm{Neu}$ roscience 140, 377-388.

Chu, Y., Jin, X., Parada, I., Pesic, A., Stevens, B., Barres, B., and Prince, D. A. (2010). Enhanced synaptic connectivity and epilepsy in C1q knockout mice. Proc. Natl. Acad. Sci. U.S.A. 107, 7975-7980.

Colton, C. A. (2009). Heterogeneity of microglial activation in the innate immune response in the brain. J. Neuroimmune Pharmacol. 4, 399-418.

Cullheim, S., and Thams, S. (2007). The microglial networks of the brain and their role in neuronal network plasticity after lesion. Brain Res. Rev. 55, 89-96.

Czeh, M., Gressens, P., and Kaindl, A. M. (2010). The yin and yang of microglia. Dev. Neurosci. 33, 199-209.

Deng, W., Aimone, J. B., and Gage, F. H. (2010). New neurons and new memories: how does adult hippocampal neurogenesis affect learning and memory? Nat. Rev. Neurosci. 11, 339-350.

Derecki, N. C., Cardani, A. N., Hui Yang, C., Quinnies, K. M., Crihfield, A., Lynch, K. L., and Kipnis, J. (2010). Regulation of learning and memory by meningeal immunity: a key role for IL-4. J. Exp. Med. 207, 1067-1080.

Dupret, D., Revest, J. M., Koehl, M., Ichas, F., De Giorgi, F., Costet, P., Abrous, D. N., and Piazza, P. V. (2008). Spatial relational memory requires hippocampal adult neurogenesis. PLoS ONE 3, e1959. doi:10.1371/journal.pone.0001959
Ekdahl, C. T., Claasen, J. H., Bonde, S., Kokaia, Z., and Lindvall, $\mathrm{O}$. (2003). Inflammation is detrimental for neurogenesis in adult brain. Proc. Natl. Acad. Sci. U.S.A. 100, 13632-13637.

Ekdahl, C. T., Kokaia, Z., and Lindvall, O. (2009). Brain inflammation and adult neurogenesis: the dual role of microglia. Neuroscience 158 , 1021-1029.

Fortin, D. A., Srivastava, T., and Soderling, T. R. (2011). Structural modulation of dendritic spines during synaptic plasticity. Neuroscientist. doi:10.1177/1073858411407206 [Epub ahead of print].

Frank-Cannon, T. C., Alto, L. T. McAlpine, F. E., and Tansey, M. G. (2009). Does neuroinflammation fan the flame in neurodegenerative diseases? Mol. Neurodegener. 4, 47.

Gascon, E., Vutskits, L., and Kiss, J. Z (2010). The role of PSA-NCAM in adult neurogenesis. Adv. Exp. Med. Biol. 663, 127-136.

Ge, S., Sailor, K. A., Ming, G. L., and Song, H. (2008). Synaptic integration and plasticity of new neurons in the adult hippocampus. J. Physiol. 586, 3759-3765.

Glass, C. K., Saijo, K., Winner, B., Marchetto, M. C., and Gage, F. H. (2010). Mechanisms underlying inflammation in neurodegeneration. Cell 140, 918-934.

Gordon, S. (2003). Alternative activation of macrophages. Nat. Rev. Immunol. 3, 23-35.

Goshen, I., Avital, A., Kreisel, T., Licht, T., Segal, M., and Yirmiya, R. (2009). Environmental enrichment restores memory functioning in mice with impaired IL-1 signaling via reinstatement of long-term potentiation and spine size enlargement. J. Neurosci. 29, 3395-3403.

Graeber, M. B. (2010). Changing face of microglia. Science 330 , 783-788.

Graeber, M. B., Li, W., and Rodriguez, M. L. (2011). Role of microglia in CNS inflammation. FEBS Lett. 585, 3798-3805.

Hattiangady, B., and Shetty, A. K. (2010). Decreased neuronal differentiation of newly generated cells underlies reduced hippocampal neurogenesis in chronic temporal lobe epilepsy. Hippocampus 20, 97-112.

Heldmann, U., Mine, Y., Kokaia, Z. Ekdahl, C. T., and Lindvall, O. (2011). Selective depletion of Mac1-expressing microglia in rat subventricular zone does not alter neurogenic response early after stroke. Exp. Neurol. 229, 391-398.
Iosif, R. E., Ekdahl, C. T., Ahlenius, H., Pronk, C. J., Bonde, S., Kokaia, Z., Jacobsen, S. E., and Lindvall, O. (2006). Tumor necrosis factor receptor 1 is a negative regulator of progenitor proliferation in adult hippocampal neurogenesis. J. Neurosci. 26, 9703-9712.

Jakubs, K., Bonde, S., Iosif, R. E., Ekdahl, C. T., Kokaia, Z., Kokaia, M., and Lindvall, O. (2008). Inflammation regulates functional integration of neurons born in adult brain. J. Neurosci. $28,12477-12488$.

Jakubs, K., Nanobashvili, A., Bonde, S., Ekdahl, C. T., Kokaia, Z., Kokaia, M. and Lindvall, O. (2006). Environment matters: synaptic properties of neurons born in the epileptic adult brain develop to reduce excitability. Neuron 52, 1047-1059.

Kettenmann, H., Hanisch, U. K., Noda, M., and Verkhratsky, A. (2011). Physiology of microglia. Physiol. Rev. 91, 461-553.

Kim, J. E., O’Sullivan, M. L., Sanchez, C. A., Hwang, M., Israel, M. A. Brennand, K., Deerinck, T. J., Goldstein, L. S., Gage, F. H., Ellisman, M. H., and Ghosh, A. (2011). Investigating synapse formation and function using human pluripotent stem cell-derived neurons. Proc. Natl. Acad. Sci. U.S.A. 108, 3005-3010.

Kipnis, J., Cohen, H., Cardon, M., Ziv, Y., and Schwartz, M. (2004). $\mathrm{T}$ cell deficiency leads to cognitive dysfunction: implications for therapeutic vaccination for schizophrenia and other psychiatric conditions. Proc. Natl. Acad. Sci. U.S.A. 101, 8180-8185.

Kubota, K., Inoue, K., Hashimoto, R., Kumamoto, N., Kosuga, A., Tatsumi, M., Kamijima, K., Kunugi, H., Iwata, N., Ozaki, N., Takeda, M., and Tohyama, M. (2009). Tumor necrosis factor receptor-associated protein 1 regulates cell adhesion and synaptic morphology via modulation of $\mathrm{N}$-cadherin expression. J. Neurochem. 110, 496-508.

Lawson, L. J., Perry, V. H., Dri, P., and Gordon, S. (1990). Heterogeneity in the distribution and morphology of microglia in the normal adult mouse brain. Neuroscience 39, 151-170.

Liu, S., Wang, J., Zhu, D., Fu, Y., Lukowiak, K., and Lu, Y. M. (2003). Generation of functional inhibitory neurons in the adult rat hippocampus. J. Neurosci. 23, 732-736.

Livneh, Y., and Mizrahi, A. (2011). Long-term changes in the morphology and synaptic distributions of adult-born neurons. J. Comp. Neurol. 519, 2212-2224. 
Luikart, B. W., Bensen, A. L., Washburn, E. K., Perederiy, J. V., Su, K. G., Li, Y., Kernie, S. G., Parada, L. F., and Westbrook, G. L. (2011). miR132 mediates the integration of newborn neurons into the adult dentate gyrus. PLoS ONE 6, e19077. doi:10.1371/journal.pone.0019077

Maggi, L., Scianni, M., Branchi, I., D'Andrea, I., Lauro, C., and Limatola, C. (2011). CX ${ }_{3}$ CR1 deficiency alters hippocampal-dependent plasticity phenomena blunting the effects of enriched environment. Front. Cell. Neurosci. 5:22. doi:10.3389/fncel.2011.00022

Magnus, T., Chan, A., Grauer, O., Toyka, K. V., and Gold, R. (2001). Microglial phagocytosis of apoptotic inflammatory $\mathrm{T}$ cells leads to down-regulation of microglial immune activation. J. Immunol. 167, 5004-5010.

Markwardt, S. J., Dieni, C. V., Wadiche, J. I., and Overstreet-Wadiche, L. (2011). Ivy/neurogliaform interneurons coordinate activity in the neurogenic niche. Nat. Neurosci. 14, 1407-1409.

Masiulis, I., Yun, S., and Eisch, A. J. (2011). The interesting interplay between interneurons and adult hippocampal neurogenesis. Mol. Neurobiol. 44, 287-302.

Massa, F., Koehl, M., Wiesner, T., Grosjean, N., Revest, J. M., Piazza, P. V., Abrous, D. N., and Oliet, S. H. (2011). Conditional reduction of adult neurogenesis impairs bidirectional hippocampal synaptic plasticity. Proc. Natl. Acad. Sci. U.S.A. 108, 6644-6649.

Mathieu, P., Piantanida, A. P., and Pitossi, F. (2010a). Chronic expression of transforming growth factorbeta enhances adult neurogenesis. Neuroimmunomodulation 17, 200-201.

Mathieu, P., Battista, D., Depino, A., Roca, V., Graciarena, M., and Pitossi, F. (2010b). The more you have, the less you get: the functional role of inflammation on neuronal differentiation of endogenous and transplanted neural stem cells in the adult brain. J. Neurochem. 112, 1368-1385.

McCoy, M. K., and Tansey, M. G. (2008). TNF signaling inhibition in the CNS: implications for normal brain function and neurodegenerative disease. J. Neuroinflammation 5, 45.

Michelucci, A., Heurtaux, T., Grandbarbe, L., Morga, E., and Heuschling, P. (2009). Characterization of the microglial phenotype under specific pro-inflammatory and antiinflammatory conditions: effects of oligomeric and fibrillar amyloidbeta. J. Neuroimmunol. 210, 3-12.

Mohapel, P., Ekdahl, C. T., and Lindvall, O. (2004). Status epilepticus severity influences the long-term outcome of neurogenesis in the adult dentate gyrus. Neurobiol. Dis. 15, 196-205.

Molina-Holgado, E., and MolinaHolgado, F. (2010). Mending the broken brain: neuroimmune interactions in neurogenesis. J. Neurochem. 114, 1277-1290.

Monje, M. L., Toda, H., and Palmer, T. D. (2003). Inflammatory blockade restores adult hippocampal neurogenesis. Science 302, 1760-1765.

Moriyama, M., Fukuhara, T., Britschgi, M., He, Y., Narasimhan, R., Villeda, S., Molina, H., Huber, B. T., Holers, M., and Wyss-Coray, T. (2011). Complement receptor 2 is expressed in neural progenitor cells and regulates adult hippocampal neurogenesis. J. Neurosci. 31, 3981-3989.

Morrens, J., Van Den Broeck, W., and Kempermann, G. (2012). Glial cells in adult neurogenesis. Glia 60, 159-174.

Mosser, D. M. (2003). The many faces of macrophage activation. J. Leukoc. Biol. 73, 209-212.

Murphy, B. L., Pun, R. Y., Yin, H., Faulkner, C. R., Loepke, A. W., and Danzer, S. C. (2011). Heterogeneous integration of adult-generated granule cells into the epileptic brain. $J$. Neurosci. 31, 105-117.

Nathan, C., and Ding, A. (2010). Nonresolving inflammation. Cell 140, 871-882.

Neher, J. J., Neniskyte, U., Zhao, J. W., Bal-Price, A., Tolkovsky, A. M., and Brown, G. C. (2011). Inhibition of microglial phagocytosis is sufficient to prevent inflammatory neuronal death. J. Immunol. 186, 4973-4983.

Neumann, J., Sauerzweig, S., Ronicke, R., Gunzer, F., Dinkel, K., Ullrich, O., Gunzer, M., and Reymann, K. G. (2008). Microglia cells protect neurons by direct engulfment of invading neutrophil granulocytes: a new mechanism of CNS immune privilege. J. Neurosci. 28, 5965-5975.

Nimmerjahn, A., Kirchhoff, F., and Helmchen, F. (2005). Resting microglial cells are highly dynamic surveillants of brain parenchyma in vivo. Science 308, 1314-1318.

O'Keeffe, G. C., and Barker, R. A. (2011). Dopamine stimulates epidermal growth factor release from adult neural precursor cells derived from the subventricular zone by a disintegrin and metalloprotease. Neuroreport 22, 956-958.

Olah, M., Biber, K., Vinet, J., and Boddeke, H. W. (2011). Microglia phenotype diversity. CNS Neurol. Disord. Drug Targets 10, 108-118.

Paolicelli, R. C., Bolasco, G., Pagani, F. Maggi, L., Scianni, M., Panzanelli, P., Giustetto, M., Ferreira, T. A. Guiducci, E., Dumas, L., Ragozzino, D., and Gross, C. T. (2011). Synaptic pruning by microglia is necessary for normal brain development. Science 333, 1456-1458.

Parent, J. M., Yu, T. W., Leibowitz, R. T., Geschwind, D. H., Sloviter, R. S., and Lowenstein, D. H. (1997). Dentate granule cell neurogenesis is increased by seizures and contributes to aberrant network reorganization in the adult rat hippocampus. J. Neurosci. 17, 3727-3738.

Perry, V. H. (2010). Contribution of systemic inflammation to chronic neurodegeneration. Acta Neuropathol. 120, 277-286.

Perry, V. H., and O'Connor, V. (2008). Clq: the perfect complement for a synaptic feast? Nat. Rev. Neurosci. 9, 807-811.

Pickering, M., Cumiskey, D., and O'Connor, J. J. (2005). Actions of TNF-alpha on glutamatergic synaptic transmission in the central nervous system. Exp. Physiol. 90, 663-670.

Polazzi, E., and Monti, B. (2010). Microglia and neuroprotection: from in vitro studies to therapeutic applications. Prog. Neurobiol. 92, 293-315.

Popa, N., Cedile, O., Pollet-Villard, X. Bagnis, C., Durbec, P., and Boucraut, J. (2011). RAE-1 is expressed in the adult subventricular zone and controls cell proliferation of neurospheres. Glia 59, 35-44.

Rahpeymai, Y., Hietala, M. A., Wilhelmsson, U., Fotheringham, A. Davies, I., Nilsson, A. K., Zwirner, J., Wetsel, R. A., Gerard, C., Pekny, M., and Pekna, M. (2006). Complement: a novel factor in basal and ischemiainduced neurogenesis. EMBO J. 25, 1364-1374.

Richerson, S., Ingram, M., Perry, D., and Stecker, M. M. (2005). Classification of the extracellular fields produced by activated neural structures. Biomed. Eng. Online 4, 53. doi:10.1186/1475-925X-4-53

Riquelme, P. A., Drapeau, E., and Doetsch, F. (2008). Brain microecologies: neural stem cell niches in the adult mammalian brain. Philos. Trans. R. Soc. Lond. B Biol. Sci. 363, 123-137.

Rivest, S. (2011). The promise of antiinflammatory therapies for CNS injuries and diseases. Expert Rev. Neurother. 11, 783-786.
Rock, K. L., Latz, E., Ontiveros, F., and Kono, H. (2010). The sterile inflammatory response. Annu. Rev. Immunol. 28, 321-342.

Ron-Harel, N., Cardon, M., and Schwartz, M. (2011). Brain homeostasis is maintained by "danger" signals stimulating a supportive immune response within the brain's borders. Brain Behav. Immun. 25, 1036-1043.

Ron-Harel, N., Segev, Y., Lewitus, G. M., Cardon, M., Ziv, Y., Netanely, D., Jacob-Hirsch, J., Amariglio, N., Rechavi, G., Domany, E., and Schwartz, M. (2008). Age-dependent spatial memory loss can be partially restored by immune activation. Rejuvenation Res. 11, 903-913.

Russo, I., Barlati, S., and Bosetti, F. (2011a). Effects of neuroinflammation on the regenerative capacity of brain stem cells. J. Neurochem. 116, 947-956.

Russo, I., Amornphimoltham, P., Weigert, R., Barlati, S., and Bosetti, F. (2011b). Cyclooxygenase-1 is involved in the inhibition of hippocampal neurogenesis after lipopolysaccharide-induced neuroinflammation. Cell Cycle 10, 2568-2573.

Sahay, A., Scobie, K. N., Hill, A. S., O'Carroll, C. M., Kheirbek, M. A., Burghardt, N. S., Fenton, A. A., Dranovsky, A., and Hen, R. (2011). Increasing adult hippocampal neurogenesis is sufficient to improve pattern separation. Nature 472, 466-470.

Sakamoto, M., Imayoshi, I., Ohtsuka, T., Yamaguchi, M., Mori, K., and Kageyama, R. (2011). Continuous neurogenesis in the adult forebrain is required for innate olfactory responses. Proc. Natl. Acad. Sci. U.S.A. 108, 8479-8484.

Schratt, G. M., Tuebing, F., Nigh, E. A., Kane, C. G., Sabatini, M. E., Kiebler, M., and Greenberg, M. E. (2006). A brain-specific microRNA regulates dendritic spine development. Nature 439, 283-289.

Seaberg, R. M., and van der Kooy, D. (2002). Adult rodent neurogenic regions: the ventricular subependyma contains neural stem cells, but the dentate gyrus contains restricted progenitors. J. Neurosci. 22, 1784-1793.

Shechter, R., London, A., Varol, C., Raposo, C., Cusimano, M., Yovel, G., Rolls, A., Mack, M., Pluchino, S., Martino, G., Jung, S., and Schwartz, M. (2009). Infiltrating bloodderived macrophages are vital cells playing an anti-inflammatory role 
in recovery from spinal cord injury in mice. PLoS Med. 6, e1000113. doi:10.1371/journal.pmed.1000113

Shinjyo, N., Stahlberg, A., Dragunow, M., Pekny, M., and Pekna, M. (2009). Complement-derived anaphylatoxin $\mathrm{C} 3 \mathrm{a}$ regulates in vitro differentiation and migration of neural progenitor cells. Stem Cells 27, 2824-2832.

Sierra, A., Encinas, J. M., Deudero, J. J., Chancey, J. H., Enikolopov, G., Overstreet-Wadiche, L. S., Tsirka, S. E., and Maletic-Savatic, M. (2010). Microglia shape adult hippocampal neurogenesis through apoptosiscoupled phagocytosis. Cell Stem Cell 7, 483-495.

Stellwagen, D., and Malenka, R. C. (2006). Synaptic scaling mediated by glial TNF-alpha. Nature 440, 1054-1059.

Stevens, B., Allen, N. J., Vazquez, L. E., Howell, G. R., Christopherson, K. S., Nouri, N., Micheva, K. D., Mehalow, A. K., Huberman, A. D., Stafford, B., Sher, A., Litke, A. M., Lambris, J. D., Smith, S. J., John, S. W., and Barres, B. A. (2007). The classical complement cascade mediates CNS synapse elimination. Cell 131, 1164-1178.

Sultan, S., Rey, N., Sacquet, J., Mandairon, N., and Didier, A. (2011). Newborn neurons in the olfactory bulb selected for long-term survival through olfactory learning are prematurely suppressed when the olfactory memory is erased. J. Neurosci. 31, 14893-14898.

Thored, P., Heldmann, U., Gomes-Leal, W., Gisler, R., Darsalia, V., Taneera, J.,
Nygren, J. M., Jacobsen, S. E., Ekdahl, C. T., Kokaia, Z., and Lindvall, O. (2009). Long-term accumulation of microglia with proneurogenic phenotype concomitant with persistent neurogenesis in adult subventricular zone after stroke. Glia 57, 835-849.

Toni, N., and Sultan, S. (2011). Synapse formation on adult-born hippocampal neurons. Eur. J. Neurosci. 33, 1062-1068.

Trapp, B. D., Wujek, J. R., Criste, G. A., Jalabi, W., Yin, X., Kidd, G. J., Stohlman, S., and Ransohoff, R. (2007). Evidence for synaptic stripping by cortical microglia. Glia 55, 360-368.

Tremblay, M. E., Lowery, R. L., and Majewska, A. K. (2010). Microglial interactions with synapses are modulated by visual experience. PLoS Biol. 8, e1000527. doi:10.1371/journal.pbio. 1000527

Tremblay, M. E., and Majewska, A. K. (2011). A role for microglia in synaptic plasticity? Commun. Integr. Biol. 4, 220-222.

Tremblay, M. E., Stevens, B., Sierra, A., Wake, H., Bessis, A., and Nimmerjahn, A. (2011). The role of microglia in the healthy brain. J. Neurosci. 31, 16064-16069.

Wake, H., Moorhouse, A. J., Jinno, S., Kohsaka, S., and Nabekura, J. (2009). Resting microglia directly monitor the functional state of synapses in vivo and determine the fate of ischemic terminals. J. Neurosci. 29, 3974-3980.

Walton, N. M., Sutter, B. M., Laywell, E. D., Levkoff, L. H., Kearns, S. M.,
Marshall, G. P. II, Scheffler, B., and Steindler, D. A. (2006). Microglia instruct subventricular zone neurogenesis. Glia 54, 815-825.

Wei, H., Zou, H., Sheikh, A. M., Malik, M., Dobkin, C., Brown, W. T., and $\mathrm{Li}, \mathrm{X}$. (2011). IL-6 is increased in the cerebellum of autistic brain and alters neural cell adhesion, migration and synaptic formation. J. Neuroinflammation 8,52 .

Whitney, N. P., Eidem, T. M., Peng, H., Huang, Y., and Zheng, J. C. (2009). Inflammation mediates varying effects in neurogenesis: relevance to the pathogenesis of brain injury and neurodegenerative disorders. J. Neurochem. 108, 1343-1359.

Wojcik-Stanaszek, L., Gregor, A., and Zalewska, T. (2011). Regulation of neurogenesis by extracellular matrix and integrins. Acta Neurobiol. Exp. (Wars) 71, 103-112

Wood, J. C., Jackson, J. S., Jakubs, K., Chapman, K. Z., Ekdahl, C. T., Kokaia, Z., Kokaia, M., and Lindvall, O. (2011). Functional integration of new hippocampal neurons following insults to the adult brain is determined by characteristics of pathological environment. Exp. Neurol. 229, 484-493.

Yirmiya, R., and Goshen, I. (2011). Immune modulation of learning, memory, neural plasticity and neurogenesis. Brain Behav. Immun. 25, 181-213.

Zhao, C., Deng, W., and Gage, F. H. (2008). Mechanisms and functional implications of adult neurogenesis. Cell 132, 645-660.
Zhao, C., Teng, E. M., Summers, R. G. Jr., Ming, G. L., and Gage, F. H. (2006). Distinct morphological stages of dentate granule neuron maturation in the adult mouse hippocampus. J. Neurosci. 26, 3-11.

Ziv, Y., Ron, N., Butovsky, O., Landa, G., Sudai, E., Greenberg, N., Cohen, H., Kipnis, J., and Schwartz, M. (2006). Immune cells contribute to the maintenance of neurogenesis and spatial learning abilities in adulthood. Nat. Neurosci. 9, 268-275.

Conflict of Interest Statement: The author declares that the research was conducted in the absence of any commercial or financial relationships that could be construed as a potential conflict of interest.

Received: 30 November 2011; paper pending published: 20 December 2011; accepted: 23 February 2012; published online: 09 March 2012.

Citation: Ekdahl CT (2012) Microglial activation - tuning and pruning adult neurogenesis. Front. Pharmacol. 3:41. doi: 10.3389/fphar.2012.00041

This article was submitted to Frontiers in Neuropharmacology, a specialty of Frontiers in Pharmacology.

Copyright (C) 2012 Ekdahl. This is an open-access article distributed under the terms of the Creative Commons Attribution Non Commercial License, which permits non-commercial use, distribution, and reproduction in other forums, provided the original authors and source are credited. 